\title{
BMJ Open Development, implementation, evaluation and scaling-up of physical activity referral schemes in Germany: protocol for a study using a co- production approach
}

Anja Weissenfels (D), Wolfgang Geidl (D), Eriselda Mino (D), Inga Naber (D), Sarah Klamroth (D), Peter Gelius (D) , Karim Abu-Omar (1) , Klaus Pfeifer (1)

To cite: Weissenfels A, Geidl W, Mino E, et al. Development, implementation, evaluation and scaling-up of physical activity referral schemes in Germany: protocol for a study using a coproduction approach. BMJ Open 2021;11:e045563. doi:10.1136/ bmjopen-2020-045563

- Prepublication history for this paper is available online. To view these files, please visit the journal online (http://dx.doi. org/10.1136/bmjopen-2020045563).

Received 06 0ctober 2020 Revised 25 February 2021 Accepted 14 March 2021
Check for updates

(C) Author(s) (or their employer(s)) 2021. Re-use permitted under CC BY-NC. No commercial re-use. See rights and permissions. Published by BMJ.

Department of Sport Science and Sport, Friedrich-AlexanderUniversität Erlangen-Nürnberg, Erlangen, Germany

Correspondence to Dr Anja Weissenfels; anja.weissenfels@fau.de

\section{ABSTRACT}

Introduction Physical activity referral schemes (PARSs) are recommended to promote physical activity (PA) among adults at risk of developing or with established non-communicable diseases (NCDs). In Germany, this kind of referral schemes has not yet been implemented systematically and nationwide. In this study protocol, we present the methodological design of a co-production research study aimed at establishing a PARS for adults with NCDs in German primary healthcare.

Methods and analysis We will employ a co-production approach consistently throughout the four project phases: (1) development of the PARS; (2) preparation period; (3) implementation and evaluation; (4) development of a strategic plan for scaling up the PARS to the national level as part of standard care. The first phase will additionally include a status quo analysis of the existing physical activity pathways nationwide as well as an overview of international PARS models. A pragmatic trial design will be used for evaluating the developed PARS. The co-production approach will involve relevant actors in the German healthcare system, namely, healthcare service providers (eg, physicians, exercise professionals), health insurance providers, exercise providers, patients' representatives, experts in the development and implementation of educational concepts, and scientists from the fields of sports science and public health.

Ethics and dissemination The project has been reviewed and approved by the ethics committee of the FriedrichAlexander-University Erlangen-Nürnberg (ethics approval number: 331_20 B). Through cooperation agreements, the stakeholders involved gave their consent to participate and were informed about the study in detail. The results of this study will be disseminated by international conference presentations and peer-reviewed publications, and if possible, a manual for the use of the PARS will be provided.

\section{INTRODUCTION}

Across the world, 1.4 billion adults do not meet the recommended level of physical activity (PA). ${ }^{1}$ This global pandemic of physical
Strengths and limitations of this study

- The study will establish a physical activity referral scheme (PARS) in primary healthcare with a co-production approach, in which all relevant stakeholders are involved in the development, implementation and evaluation of the PARS.

- The co-production approach facilitates the transfer to standard care and considers individual barriers and facilitating factors.

- Developing a scaling-up strategy at the end of the project will help facilitate nationwide dissemination of the PARS into the German primary healthcare system.

- The science-driven approach to initiating the coproduction study may hamper true co-production in the development and implementation of PARSs.

- End users were not directly involved in the coproduction process.

inactivity $^{2}$ contributes to the increasing rates of non-communicable diseases (NCDs) and premature death ${ }^{3}$ and creates an additional economic cost of at least US $\$ 67.5$ billion per year. ${ }^{4}$ Accordingly, PA promotion is a global challenge. To support nations in their fight against physical inactivity, WHO has developed the Global Action Plan on Physical Activity 2018-2030. ${ }^{5}$ This plan contains a system-based approach, including ideas for creating active societies, active environments, active systems and, of course, active people. The proposed actions for reaching these objectives include a multitude of different interventions, for example, conducting communication campaigns (active societies), strengthening access to good-quality public and green open spaces (active environments), and enhancing national data systems for regular population surveillance of 
PA (active systems). One proposed action to create more active people is the integration of physical activity referral schemes (PARSs), meaning that physicians prescribe PA or exercise for their patients with long-term NCDs such as cardiovascular disease, diabetes, cancer and mental illness, or after stroke. Depending on the authors and countries, the terminology varies between 'exercise referral scheme' (ERS) and 'physical activity referral scheme', although these terms are also often used simultaneously. Whereas ERSs are more closely related to exercise classes, PA promotion interventions aim more at individual behaviour change. ${ }^{6}$

Integrating referral schemes into healthcare is an evidence-based approach for PA promotion, ${ }^{8}$ especially in adults with NCDs. ${ }^{9}$ The high potential of referral schemes for promoting PA is mainly related to two aspects. First, PARSs prioritise and address one of the least active populations, namely adults with NCDs. ${ }^{10} 11$ Second, incorporating $\mathrm{PA}$ promotion into the primary healthcare system effectively reaches those who need it; many people with NCDs are familiar with primary care because they are treated at least once per year by a general physician, and patients trust medical staff as credible. ${ }^{12}$ Physicians can already integrate patient assessment on PA levels, give brief advice or short counselling on increasing PA, and then refer to appropriate supervised support for PA. Internationally, some countries (eg, New Zealand, Denmark, Sweden, Norway, UK) have already successfully integrated PA promotion interventions into their primary healthcare systems. Evidence suggests the modest but positive effect of PARSs on PA level ${ }^{81314}$ and other healthrelated outcomes, including reduced risk of all-cause mortality, cardiovascular mortality and cardiovascular events. ${ }^{15}$ Pooled relative risks in systematic reviews have shown similar degrees of effectiveness on the proportion of individuals engaging in moderate PA, ranging from 1.12 (95\% CI 1.04 to 1.20$),{ }^{16} 1.16$ (95\% CI 1.03 to $1.30)^{17}$ up to 1.20 (95\% CI 1.06 to 1.35$) .{ }^{18}$ Participation in a PARS is associated with an increased proportion of persons achieving at least $90-150$ min of moderate PA per week ${ }^{18}$ when compared with standard care. ${ }^{16}$ These benefits seem to result from the effective use of healthcare resources, ${ }^{16}$ making PARSs a good alternative to interventions such as PA advice. ${ }^{19}$ However, authors have highlighted uncertainty in the current evidence base about cost-effectiveness, ${ }^{18}$ long-term effects on PA, ${ }^{17} 18$ and other clinical and psychological outcomes. ${ }^{17}$ Therefore, further evaluation of the PARS approach for persons at risk or with underlying NCDs remains an important part of the research agenda. ${ }^{20}$

In Germany, previous attempts to establish PARSs within the healthcare system have been successful regionally but not nationwide. The best-known PA promotion programme so far was 'Rezept für Bewegung' (Physical activity on prescription) ${ }^{21}$ However, it has recently been reported that only a small portion of general practitioners is aware of this programme. ${ }^{22}$ Additional barriers for a nationwide dissemination of 'Rezept für Bewegung' include that financial reimbursement is not possible, the effectiveness of its PA promotion is doubted, and the referral to sports and exercise programmes associated with the prescription is difficult due to few or unsuitable offers. ${ }^{12}$ Lack of time, missing manuals and a lack of knowledge about concrete implementation are also inhibiting factors. ${ }^{22}$ Although PARSs are explicitly recommended in the recently developed National Recommendations on PA and PA promotion in adults with NCDs, ${ }^{9}$ they have not yet been systematically anchored in the German primary care system.

\section{Objectives}

Therefore, the aim of the project BewegtVersorgt is the co-production development, implementation and evaluation, as well as the dissemination, of a physician-initiated PARS for people with NCDs in the context of primary healthcare in Germany.

\section{METHODS}

The study described in this protocol employs various methods to establish a PARS for people with NCDs in the German healthcare system. This protocol is based on the Standard Protocol Items: Recommendations for Interventional Trials (SPIRIT) ${ }^{23}$ However, due to the co-production approach, not all the information on the SPIRIT checklist can be given in this phase, so a separate protocol will be established for the pragmatic trial after the second phase (see table 1). Our pragmatic trial will be conducted and reported in accordance with the reporting guidelines provided in the CONSORT 2010 statement, ${ }^{24}$ taking into account the CONSORT extension for pragmatic trials. ${ }^{25}$ Due to the various methods used in this study, guidelines for the qualitative measurement procedures (Standards for Reporting Qualitative Research-SRQR ${ }^{26}$ ) and for the systematic review (Preferred Reporting Items for Systematic Reviews and Meta-Analyses-PRISMA ${ }^{27}$ ) will also be considered.

\section{Setting and participants}

BewegtVersorgt refers to primary care in the German healthcare system. The innovative idea of this research is to involve relevant actors in the healthcare system in a co-production approach to develop, implement and evaluate a PARS. We use the term co-production as defined by Hickey. It includes the participation of multiple stakeholders (eg, researchers, practitioners, the public), who work together and share power and responsibility for the whole project. ${ }^{28}$ The main idea of the project is to include relevant stakeholders of the German healthcare system and sport system who represent different areas of the healthcare system and are experts in their respective fields. In cooperation with the research team $(n=8)$ from the Department of Sport Science and Sport (DSS) at Friedrich-Alexander-University Erlangen-Nürnberg (FAU), the following stakeholders and representatives 
Table 1 Study phases, research questions and methodology

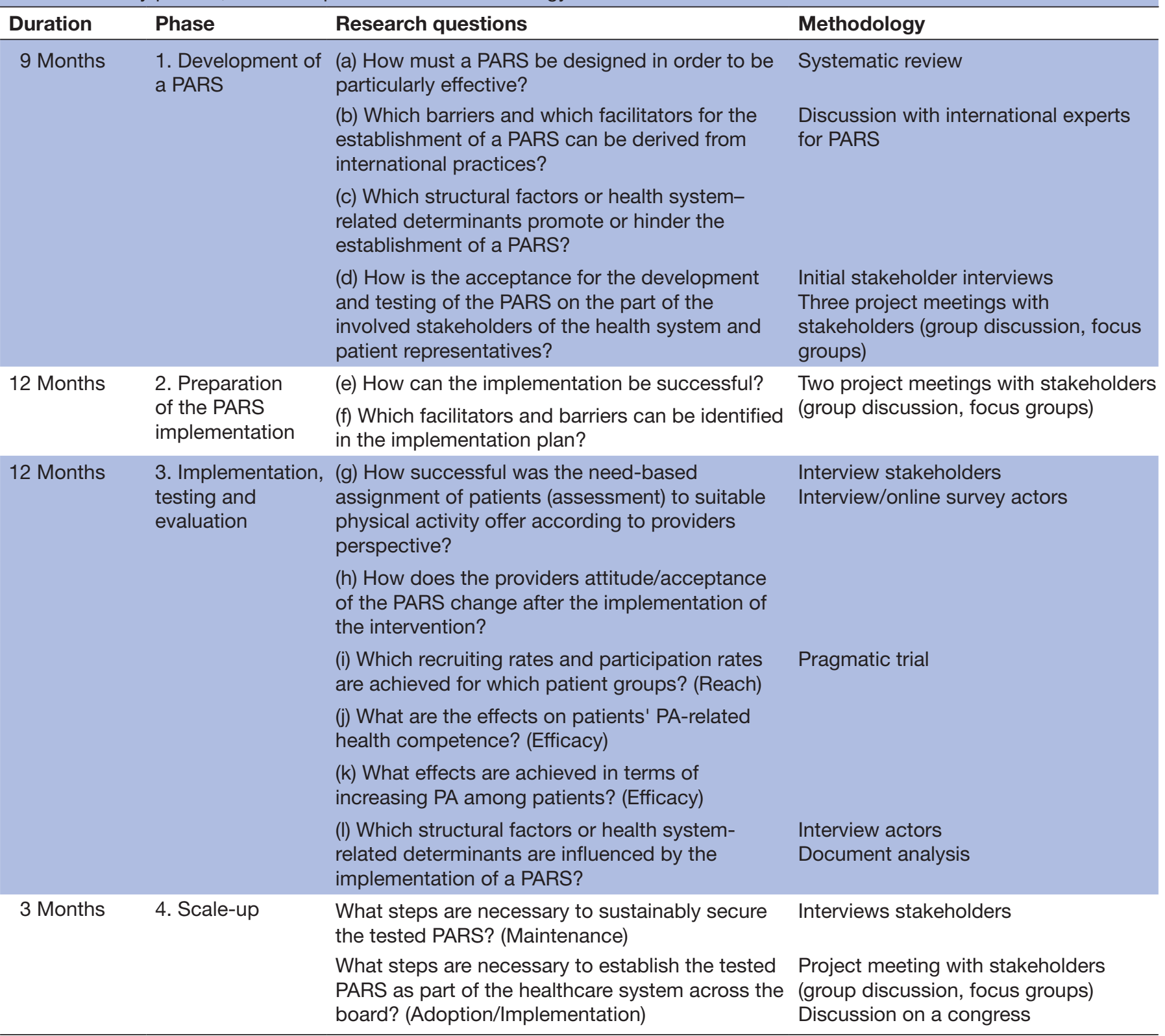

PA, physical activity; PARS, physical activity referral scheme.

from 12 important organisations will participate in the co-production approach ('co-production team'):

- Five local general practitioners and sport physicians acting as representatives of three different physician associations of Bavaria (Bayerischer Hausärzteverband, Bayerischer Sportärzteverband, Bayerischer Landesärzteverband);

- Four representatives of two of the largest Bavarian healthcare insurance companies (AOK Bayern - Die Gesundheitskasse, DAK-Gesundheit (Landesverband Bayern));

- Three representatives of three associations for exercise professionals and physical therapists (Deutscher Verband für Gesundheitssport und Sporttherapie,
Bundesverband selbstständiger Physiotherapeuten, VDB-Physiotherapieverband);

- Two representatives of the German Olympic Sports Association as the umbrella organisation of the German sport system (Deutscher Olympischer Sportbund);

- Two representatives of a centre for patient education and PA promotion (Zentrum Patientenschulung und Gesundheitsförderung); and

- Potential end users of the PARS, patients with NCDs, represented by two persons acting as representatives of two large patient organisations (Deutsche DiabetesHilfe-Menschen mit Diabetes e.V., Deutsche Rheuma-Liga Landesverband Bayern e.V.). 
The research team of DSS has extensive experience with co-production approaches in the field of behavioural PA promotion and public health through other projects (eg, BIG,${ }^{29}$ Capital4Health, ${ }^{30}$ PArC-AVE ${ }^{31}$ ). The team will adopt the role of transferring knowledge, providing scientific evidence, and coordinating the entire project.

The target group for which the PARS is to be developed and tested comprises persons with NCDs who are physically inactive. As this group is very broad, and due to funder requirements and practical feasibility, the target group will be further specified (health conditions, age groups, local/regional demand) within the co-production process.

The 12 stakeholder organisations, both regional and national, have each expressed their willingness to participate in the project through a written cooperation agreement. Regarding the potential scaling-up of the PARS, if successfully implemented and proven effective within the project, it is anticipated that the co-production approach with the relevant stakeholders will also ease the potential transfer to standard care.

\section{Study design and phases}

The project BewegtVersorgt is based on various elements of implementation research, including theories of organisational readiness ${ }^{32}$ and the dissemination of innovations. ${ }^{33}$ Normalisation process theory will be used to develop a concept for the continuity of the PARS. ${ }^{34}$ For the development of an adequate study design, the project will be guided by the categories provided by Curran $e t$ $a l .{ }^{35}$ Regarding their description of hybrid designs in a continuum between effectiveness and implementation of research methods, our study can be classified as hybrid type II. ${ }^{36}$ This type describes a design that simultaneously tests the (clinical) effectiveness of both the intervention and the implementation strategy.

The chosen 'co-production' approach ${ }^{28} 36$ provides the best possibility for linking the different perspectives and needs of patients, funders and service providers in a care setting with the scientific evidence on PA for people with NCDs. Participation is an integral part of health promotion in general and is implemented in BewegtVersorgt through a high degree of cooperation and co-determination. Within the stage model, according to Wright et $a l,{ }^{37}$ to assess the extent of participation (degree of decision-making power), one can basically distinguish between preliminary stages of 'participation' (information, consultation, involvement) and 'genuine participation' (co-determination, partial decision-making power, decision-making authority). ${ }^{37}$ In BewegtVersorgt, all stakeholders are involved in central decision-making processes in project planning, implementation and evaluation in the sense of 'genuine participation'.

The entire project is planned from June 2019 until May 2022 and is divided into four project phases (see table 1). In the first (06/2019-02/2020) and second (03/2020-02/2021) phases, the PARS will be developed and prepared for implementation. In the third phase of the project $(03 / 2021-02 / 2022)$, the planned PARS will be implemented and evaluated in a regional model project. Based on the evaluation results, a scaling-up strategy will be developed in the last phase of the study $(03 / 2022-05 / 2022)$. In what follows, the various phases, as well as the method of data collection, are explained in more detail. Collaboration between the research team and the stakeholders is part of each of the four phases. The research questions of the following four phases are listed in table 1.

\section{Phase 1: developing a PARS}

To learn from international experiences of PARSs, the research team will conduct a systematic review with the aim of identifying core intervention components that promote PARS effectiveness. We will seek to analyse the design of international models (eg, New Zealand, Sweden) and identify important key elements that contribute to their effectiveness. In this context, we will also investigate challenges of other referral schemes (eg, low adherence, lack of long-term commitment) and strategies for minimising them. This will be registered at PROSPERO.

The research team will also conduct interviews or joint discussions with international experts in PA promotion (especially PA promotion). The involvement of international experts will not be limited to the first phase of the project but can also occur at other points of the project when appropriate.

In parallel, the research team will conduct individual semi-structured interviews with representatives of the 12 aforementioned stakeholder organisations. Semistructured interviews are appropriate because they will allow the stakeholders to express their ideas for a potential PARS in the German healthcare system without potential role-dependent or hierarchy-dependent influences and address stakeholder needs and expectations.

To measure the acceptability of the PARS development process and evaluation from the stakeholder perspective, co-production meetings are planned. All representatives of the 12 stakeholder organisations will be invited to three co-production meetings that will take place during the first project phase. These meetings will be organised by the DSS research team, which will plan the content and act as a neutral moderator. The research team will present the results of the preliminary work to the participating stakeholders, laying the ground for the co-production of a PARS model for Germany. During the meetings, a focus group format, expert input and plenary discussions will be used depending on the needs and stages of progress. Protocols and notes of these events will be taken and transcribed by two persons not involved in the development process to allow for unbiased documentation and to provide results to all members of the co-production process.

\section{Phase 2: preparation of the PARS implementation}

In the preparatory phase for the implementation of the developed PARS, the question of how the implementation 
can be successful among the participating actors will be investigated. In addition, success factors and barriers are to be identified through interviews, focus groups and document analysis (see table 1). For the successful implementation of the PARS in standard care, communication among the stakeholders is particularly important in this project phase, as they cover different areas of expertise. For example, healthcare insurance providers will help in preparing a contract, and the entire team, consisting of the research team and the representatives of the 12 stakeholder organisations, will help decide on important components of the pragmatic trial (eg, target group, intervention content, study design). Two joint meetings are planned for this purpose, where discipline-specific topics will also be clarified through bilateral and multilateral discussions. The joint creation of an implementation plan and decision-making processes in the preparation phase will reflect the co-production approach. The development of relevant outcomes (primary, secondary) will also be based on a joint decision-making process. Due to the stakeholders' different perspectives, relevant outcomes may vary and should be queried via an online survey.

Compared with an exploratory trial, which takes place under optimal standardised conditions, a pragmatic trial is very close to usual care conditions. The PRECIS tool supports the choice of a pragmatic trial over an exploratory one and enables the balancing of internal and external validity in the design of the study. ${ }^{38}$ Although PRECIS is a widely used and helpful tool, studies have identified weaknesses in the quality criteria (eg, validity, inter-rater reliability), as well as a lack of a scoring system and expandable guidance, resulting in the development of an adapted tool called PRECIS II. ${ }^{39}$ The present study will use these tools in the design of the pragmatic trial.

\section{Phase 3: implementation, testing and evaluation}

This phase will include the exemplary implementation, testing and evaluation of the developed PARS. The research questions are listed in table 1.

As the study is designed to be a co-production process in which all participants also decide on components of the pragmatic trial, no specific information about intervention arms, target group, randomisation, sample size calculation or statistical analysis can be given in advance. These will be presented in a separate study protocol (based on SPIRIT) and will be registered on a clinical trial register (ClinicalTrials.gov) at a later date. The main characteristic of co-production planning models is unpredictability, which is why previous steering or influencing would have a negative impact on the process.

The overall aim of the PARS is to increase PA among inactive patients with NCDs in the German healthcare setting. The funding organisation has already established specifications regarding the time frame of the pragmatic trial and region of testing. The PARS will be tested in the Nuremberg Metropolitan Region, mainly in the cities of Erlangen, Nuremberg and Fürth. Different actors in the healthcare system, such as general practitioners, physical therapists, sport organisations or fitness clubs, will be recruited to participate in the pragmatic trial. To test the effects of the PARS, three measurements are planned at baseline, 12 weeks and 24 weeks of follow-up. Preferably, we will integrate a control group and conduct a randomised controlled trial or a cluster-randomised controlled trial. This design will follow the CONSORT extension for pragmatic trials. ${ }^{25}$ The decision regarding the study design is based on the joint decision-making process and external contextual factors, such as financial reimbursement from health insurance providers.

In addition, qualitative and quantitative data for evaluating the implementation and efficacy of the intervention will be analysed with adapted mixed methods. Through the co-production approach, all stakeholders will have the opportunity to define further impact dimensions for the evaluation as well as possible outcomes.

In addition to testing the PARS, this phase will include an evaluation plan prepared jointly with the stakeholders. The evaluation plan will be based on the RE-AIM framework for evaluating the public health impact of an intervention according to five dimensions: reach, effectiveness, adoption, implementation and maintenance. ${ }^{40}$ In this way, effectiveness dimensions can be mapped at both the patient level (eg, participation in prescribed exercise programmes) and the system level (eg, changes in work processes, public health impact, cost-effectiveness). Thus, decisions about outcomes and measurement methods will emerge during the collaborative process and cannot be reported in detail at present. However, it can be assumed that they may be diverse due to the different stakeholder interests (eg, improve PA level, health changes, costeffectiveness). Due to a possible contractual integration of the pragmatic trial into a 'model project' according to \$64 SGB V (German Social Security Code), an independent external evaluation is also necessary for dealing, for example, with health economic issues. In particular, the use of the RE-AIM framework, in addition to the co-production approach, contributes significantly to the sustainable implementation of the developed PARS. To identify barriers and success factors to implementing the PARS intervention and also to evaluate the co-production process, both open and structured or semi-structured interviews or online surveys will be used. For a further scaling-up strategy and the evaluation of the project, written overviews of the entire exchange, contents, success and inhibition factors will be documented in order to enable a later follow-up of all events that happened.

\section{Phase 4: scale-up}

As Koorts et al report, the number of effective interventions to increase PA has been growing in recent years, but only a small number of them are transferred to standard care. ${ }^{41}$ By using a pragmatic trial according to a hybrid type II design ${ }^{35}$ the developed PARS will be simultaneously tested for effectiveness and implementation in standard care. The PRACTIS (PRACTical Planning for 
Implementation and Scale-up) guide ${ }^{41}$ will be used as a helpful tool to inform the methodological process in this project phase. In the final phase of the project, a strategy for the long-term maintenance and scaling-up of the PARS will be developed. This phase will involve answering the questions, "What steps are necessary to sustainably secure the tested PARS?" (Maintenance) and "What steps are necessary to establish the tested PARS as part of the healthcare system across the board?" (Adoption/ Implementation) (see table 1). The focus is on the joint establishment of a concept for the transfer of the PARS to other regions. This will be coordinated by the research team with all 12 stakeholder organisations involved. If stakeholders or actors of the PARS have reported problems in the previous phases of implementation, qualitative interviews will be conducted with these stakeholders and actors to better understand possible barriers to transfer. The final transfer concept will be presented at a workshop and discussed with nationwide representatives of the healthcare system from science as well as from politics and practice. If a transfer to national standard care does not work, a transfer to other model regions or the expansion of the target group can be considered.

\section{Patient and public involvement}

This study involves relevant stakeholders of the German healthcare system in key decision-making processes in the development, implementation and evaluation of the PARS intervention. These stakeholders include representatives of healthcare providers (general practitioners and exercise and physical therapists), health insurance companies, patient organisations (as end-user representatives) and sport organisations. This procedure is intended to promote co-production and early acceptance and facilitate later dissemination and scaling-up. Stakeholders will be involved through regular meetings with all project partners as well as discussion rounds, meetings between individual institutions, status updates via email and online surveys, for example, on possible outcomes of the pragmatic trial. The aforementioned representatives were only partly involved in the overall design of the project by providing initial information about the planned approach and providing comments after they had been contacted for letters of intent in advance of the submission of the research proposal. Due to the long project duration of 3 years and the difficult organisational involvement of patients, the end users, patients with NCDs, were represented by two large organisations representing the patient perspective (Deutsche Diabetes-Hilfe-Menschen mit Diabetes e.V. and Deutsche Rheuma-Liga Landesverband Bayern e.V.). When selecting the stakeholders, special attention was paid to explicitly involve persons who have experience through a disease and can, therefore, represent the patients' perspective.

\section{Data management}

All data will be transcribed, pseudonymised, recorded, archived and evaluated by project staff using qualitative research methods (eg, transcription and coding of structured interviews via MAXQDA) and quantitative methods (descriptive and inferential statistics via, for example, SPSS, R). The data will be stored securely, and only direct project members of the DSS will have access to them. The deletion of all collected data is planned after the legal retention period of 10 years, beginning with the end of the project duration (31 May 2022).

\section{Ethics and dissemination}

The ethics approval has been obtained for the BewegtVersorgt-project at the responsible institution at FAU (ethics approval number: 331_20 B).

The study follows the rules of the Declaration of Helsinki. Data will be published only in pseudo-anonymised form, so that no assignment to individual persons can take place. Data protection will be respected during the entire project duration, and the participating stakeholders were informed in advance about the study project. By means of a cooperation agreement, they have given their written informed consent to participate in the study. They may revoke this consent at any time without giving reasons. It is intended that the results of the study will be published in peer-reviewed journals and presented at international conferences. Furthermore, it is anticipated that a manual for the developed PARS and an educational concept will be made available to the actors involved (eg, physiotherapists, exercise therapists, and health professionals in primary care).

\section{DISCUSSION}

This project will establish a PARS for adults with NCDs in German primary healthcare. The co-production approach chosen will increase the chances of successfully developing, implementing and evaluating the PARS. The scaling-up strategy forms the basis for a national dissemination of the PARS into the German primary healthcare system. Due to the joint approach between scientists and actors from the healthcare system, details about the PARS, testing (study design, intervention content, target group) and evaluation (outcomes, measurement methods) cannot be presented in detail at this time. Details of the upcoming pragmatic trial will be described in an additional study protocol; this trial will also be registered at ClinicalTrials.gov. Following the example of recent study protocols ${ }^{42-44}$ or articles, ${ }^{45}$ this study protocol aims to present the entire process of a complex, multiphase co-production project. The joint development process in particular plays an important role in the acceptance of new services, such as PARSs, by all stakeholders of the healthcare system. The present study protocol follows recent calls for more precise reporting of such studies $\left(\mathrm{eg},{ }^{4044-46}\right)$ and for providing a thorough description of the conducted activities, the underlying logic and steps of the co-production process. Recent papers on standards of reporting have issued the same call (CERT, ${ }^{46} \mathrm{TIDieR}^{47}$ ). 
Thus, we think this protocol helps to fill this gap also by providing the basis for further publications coming from our trial.

Acknowledgements We would like to thank the following partners for their cooperation in the project BewegtVersorgt: Bayerischer Landesärztekammer (BLÄK), Bayerischer Hausärzteverband (BHÄV), Hausärzte Erlangen und Umgebung e.V., Bayerischer Sportärzteverband (BSÄV), Deutscher Verband für Gesundheitssport und Sporttherapie (DVGS), Bundesverband selbstständiger Physiotherapeuten (IFK e.V.), VDB-Physiotherapieverband, Deutscher Olympischer Sportbund (DOSB), Deutsche Diabetes-Hilfe-Menschen mit Diabetes e.V. (DDH-M), Deutsche Rheuma-Liga Landesverband Bayern e.V., AOK Bayern—Die Gesundheitskasse, DAK-Gesundheit (Landesverband Bayern) and Zentrum Patientenschulung und Gesundheitsförderung (ZePG e.V.).

Contributors KP had the initial idea for this project and submitted the funding application for the study together with WG, KA-0 and PG. AW and SK coordinate the project. AW, WG, EM, IN, SK, PG, KA-0 and KP designed the study and will support the co-production process from a scientific perspective and moderate the decisionmaking process. AW, SK, EM and IN will monitor the co-production process as well as select and analyse data. AW and WG prepared the draft of this study protocol. All authors contributed substantially to the drafting of the paper and its revisions. All authors have read and approved the final manuscript.

Funding The study is funded by the Federal Ministry of Health based on a resolution of the German 'Bundestag' by the federal government (grant number: ZMV I 1 - 2519FSB109).

Competing interests None declared.

Patient and public involvement Patients and/or the public were involved in the design, or conduct, or reporting, or dissemination plans of this research. Refer to the Methods section for further details.

Patient consent for publication Not required.

Provenance and peer review Not commissioned; externally peer reviewed.

Open access This is an open access article distributed in accordance with the Creative Commons Attribution Non Commercial (CC BY-NC 4.0) license, which permits others to distribute, remix, adapt, build upon this work non-commercially, and license their derivative works on different terms, provided the original work is properly cited, appropriate credit is given, any changes made indicated, and the use is non-commercial. See: http://creativecommons.org/licenses/by-nc/4.0/.

\section{ORCID iDs}

Anja Weissenfels http://orcid.org/0000-0002-3271-4935

Wolfgang Geidl http://orcid.org/0000-0002-7106-7742

Eriselda Mino http://orcid.org/0000-0002-1885-0009

Inga Naber http://orcid.org/0000-0003-2766-2912

Sarah Klamroth http://orcid.org/0000-0002-1302-1842

Peter Gelius http://orcid.org/0000-0002-4120-4996

Karim Abu-Omar http://orcid.org/0000-0002-8686-7013

Klaus Pfeifer http://orcid.org/0000-0003-2418-373X

\section{REFERENCES}

1 Guthold R, Stevens GA, Riley LM, et al. Worldwide trends in insufficient physical activity from 2001 to 2016: a pooled analysis of 358 population-based surveys with 1.9 million participants. Lancet Glob Health 2018;6:e1077-86.

2 Kohl HW, Craig CL, Lambert EV, et al. The pandemic of physical inactivity: global action for public health. Lancet 2012;380:294-305.

3 Lee I-M, Shiroma EJ, Lobelo F, et al. Effect of physical inactivity on major non-communicable diseases worldwide: an analysis of burden of disease and life expectancy. Lancet 2012;380:219-29.

4 Ding D, Lawson KD, Kolbe-Alexander TL, et al. The economic burden of physical inactivity: a global analysis of major non-communicable diseases. Lancet 2016;388:1311-24 http://www.sciencedirect.com/ science/article/pii/S014067361630383X

5 World Health Organization. Global action plan on physical activity 2018-2030: more active people for a healtier world. Geneva World Health Organization; 2018.

6 Buckley BJ, Thijssen DH, Murphy RC, et al. Pragmatic evaluation of a coproduced physical activity referral scheme: a UK quasiexperimental study. BMJ Open 2020;10:e034580.
7 Dugdill L, Graham RC, McNair F. Exercise referral: the public health panacea for physical activity promotion? A critical perspective of exercise referral schemes; their development and evaluation. Ergonomics 2005;48:1390-410.

8 Onerup A, Arvidsson D, Blomqvist Åse, et al. Physical activity on prescription in accordance with the Swedish model increases physical activity: a systematic review. Br J Sports Med 2019;53:383-8.

9 Geidl W, Abu-Omar K, Weege M, et al. German recommendations for physical activity and physical activity promotion in adults with noncommunicable diseases. Int J Behav Nutr Phys Act 2020;17:12.

10 Brawner CA, Churilla JR, Keteyian SJ. Prevalence of physical activity is lower among individuals with chronic disease. Med Sci Sports Exerc 2016;48:1062-7.

11 Barker J, Smith Byrne K, Doherty A, et al. Physical activity of UK adults with chronic disease: cross-sectional analysis of accelerometer-measured physical activity in 96706 UK Biobank participants. Int J Epidemiol 2019;48:1386-74.

12 Kleinert J, Belz J, Zepp C. Aus der Arztpraxis in den Sportverein?: Herausforderung an eine ärztliche Präventionsempfehlung zur Veränderung des Bewegungsverhaltens. Köln Deutsche Sporthochschule; 2016.

13 Hamlin MJ, Yule E, Elliot CA, et al. Long-term effectiveness of the New Zealand Green Prescription primary health care exercise initiative. Public Health 2016;140:102-8.

14 Arsenijevic J, Groot W. Physical activity on prescription schemes (PARS): do programme characteristics influence effectiveness? Results of a systematic review and meta-analyses. BMJ Open 2017;7:e012156.

15 Journath G, Hammar N, Vikström M, et al. A Swedish primary healthcare prevention programme focusing on promotion of physical activity and a healthy lifestyle reduced cardiovascular events and mortality: 22-year follow-up of 5761 study participants and a reference group. Br J Sports Med 2020;54:1294-9.

16 Campbell F, Holmes M, Everson-Hock E, et al. A systematic review and economic evaluation of exercise referral schemes in primary care: a short report. Health Technol Assess 2015;19:1-110.

17 Pavey TG, Taylor AH, Fox KR, et al. Effect of exercise referral schemes in primary care on physical activity and improving health outcomes: systematic review and meta-analysis. BMJ 2011;343:d6462.

18 Williams NH, Hendry M, France B, et al. Effectiveness of exercisereferral schemes to promote physical activity in adults: systematic review. Br J Gen Pract 2007;57:979-86.

19 Elley CR, Garrett S, Rose SB, et al. Cost-effectiveness of exercise on prescription with telephone support among women in general practice over 2 years. Br J Sports Med 2011;45:1223-9.

20 National Institute for Health and Care Excellence. Physical activity: exercise referral schemes (PH54). London NICE; 2014.

21 Löllgen H, Wismach J, Kunstmann W. Das Rezept für Bewegung - Einsatzmöglichkeiten für Arzt und Patient: [Exercise prescription for health - Benefit for practitioner and patients]. Klinikarzt 2013:42:416-20.

22 Curbach J, Apfelbacher C, Knoll A, et al. Physicians' perspectives on implementing the prevention scheme "Physical Activity on Prescription": Results of a survey in Bavaria. Z Evid Fortbild Qual Gesundhwes 2018;131-132:66-72.

23 Chan A-W, Tetzlaff JM, Altman DG, et al. SPIRIT 2013 statement: defining standard protocol items for clinical trials. Ann Intern Med 2013;158:200-7.

24 Schulz KF, Altman DG, Moher D, et al. CONSORT 2010 statement: updated guidelines for reporting parallel group randomised trials. BMJ 2010;340:C332.

25 Zwarenstein M, Treweek S, Gagnier JJ, et al. Improving the reporting of pragmatic trials: an extension of the CONSORT statement. BMJ 2008;337:a2390.

26 O'Brien BC, Harris IB, Beckman TJ, et al. Standards for reporting qualitative research: a synthesis of recommendations. Acad Med 2014;89:1245-51.

27 Moher D, Liberati A, Tetzlaff J, et al. Preferred reporting items for systematic reviews and meta-analyses: the PRISMA statement. BM 2009;339:b2535.

28 Hickey DG. The potential for coproduction to add value to research. Health Expect 2018;21:693-4.

29 Rütten A, Frahsa A, Abel T, et al. Co-producing active lifestyles as whole-system-approach: theory, intervention and knowledge-toaction implications. Health Promot Int 2019;34:47-59.

30 Gelius P, Brandl-Bredenbeck HP, Hassel H. Kooperative Planung von Maßnahmen zur Bewegungsförderung Neue Wege zur Erweiterung von Handlungsmöglichkeiten - Ergebnisse aus dem Forschungsverbund Capital4Health. Bundesgesundheitsblatt Gesundheitsforschung Gesundheitsschutz 2021;64:187-98. 
31 Popp J, Carl J, Grüne E, et al. Physical activity promotion in German vocational education: does capacity building work? Health Promot Int 2020;35:1577-89.

32 Weiner BJ. A theory of organizational readiness for change. Implement Sci 2009;4:67.

33 Greenhalgh T, Robert G, Macfarlane F, et al. Diffusion of innovations in service organizations: systematic review and recommendations. Milbank Q 2004;82:581-629.

34 May C, Finch T, Mair F, et al. Understanding the implementation of complex interventions in health care: the normalization process model. BMC Health Serv Res 2007;7.

35 Curran GM, Bauer M, Mittman B, et al. Effectiveness-implementation hybrid designs: combining elements of clinical effectiveness and implementation research to enhance public health impact. Med Care 2012;50:217-26.

36 Batalden M, Batalden P, Margolis P, et al. Coproduction of healthcare service. BMJ Qual Saf 2016;25:509-17.

37 Wright MT, Block M, von Unger $\mathrm{H}$. [Participation in the cooperation between target group, project and sponsor]. Gesundheitswesen 2008;70:748-54.

38 Thorpe KE, Zwarenstein M, Oxman AD, et al. A pragmaticexplanatory continuum indicator summary (PRECIS): a tool to help trial designers. CMAJ 2009;180:E47-57.

39 Loudon K, Treweek S, Sullivan F, et al. The PRECIS-2 tool: designing trials that are fit for purpose. BMJ 2015;350:h2147.

40 Glasgow RE, Vogt TM, Boles SM. Evaluating the public health impact of health promotion interventions: the RE-AIM framework. Am J Public Health 1999;89:1322-7.
41 Koorts H, Eakin E, Estabrooks P, et al. Implementation and scale up of population physical activity interventions for clinical and community settings: the PRACTIS guide. Int J Behav Nutr Phys Act 2018;15:51.

42 Steele Gray C, Tang T, Armas A, et al. Building a digital bridge to support patient-centered care transitions from hospital to home for older adults with complex care needs: protocol for a codesign, implementation, and evaluation study. JMIR Res Protoc 2020;9:e20220.

43 Papoutsi C, Hargreaves D, Colligan G, et al. Group clinics for young adults with diabetes in an ethnically diverse, socioeconomically deprived setting (TOGETHER study): protocol for a realist review, codesign and mixed methods, participatory evaluation of a new care model. BMJ Open 2017;7:e017363.

44 Brown MC, Araújo-Soares V, Skinner R, et al. Using qualitative and co-design methods to inform the development of an intervention to support and improve physical activity in childhood cancer survivors: a study protocol for BEing Active after ChildhOod caNcer (BEACON) BMJ Open 2020;10:e041073.

45 Buckley BJR, Thijssen DHJ, Murphy RC, et al. Making a move in exercise referral: co-development of a physical activity referral scheme. J Public Health 2018;40:e586-93 https://pubmed.ncbi.nlm. nih.gov/29688551/

46 Slade SC, Dionne CE, Underwood M, et al. Consensus on Exercise Reporting Template (CERT): explanation and elaboration statement. Br J Sports Med 2016;50:1428-37.

47 Hoffmann TC, Glasziou PP, Boutron I, et al. Better reporting of interventions: template for intervention description and replication (TIDieR) checklist and guide. BMJ 2014;348:g1687. 